\title{
Kovacs effect in facilitated spin models of strong and fragile glasses
}

\author{
Jeferson J. Arenzon* \\ Instituto de Física, Universidade Federal do Rio Grande do Sul \\ CP 15051, 91501-970 Porto Alegre RS, Brazit

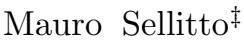 \\ The Abdus Salam International Centre for Theoretical Physics \\ Strada Costiera 11, 34100 Trieste, Italy
}

\begin{abstract}
We investigate the Kovacs (or crossover) effect in facilitated $f$-spin models of glassy dynamics. Although the Kovacs hump shows a behavior qualitatively similar for all cases we have examined (irrespective of the facilitation parameter $f$ and the spatial dimension $d$ ), we find that the dependence of the Kovacs peak time on the temperature of the second quench allows to distinguish among different microscopic mechanisms responsible for the glassy relaxation (e.g. cooperative vs defect diffusion). We also analyze the inherent structure dynamics underlying the Kovacs protocol, and find that the class of facilitated spin models with $d>1$ and $f>1$ shows features resembling those obtained recently in a realistic model of fragile glass forming liquid.
\end{abstract}

PACS numbers: $61.20 . \mathrm{Lc}, 64.70 . \mathrm{Pf}, 75.10 . \mathrm{Nr}$

\section{Introduction}

There is much interest in understanding how the out of equilibrium properties of glassy materials can be described within a statistical mechanics framework. At low temperatures, the exceedingly long relaxation time prevents such materials from reaching thermal equilibrium on the accessible time scales, and makes hard to establish a connection with the thermodynamic phase behavior [1]. In particular, several questions about the nature of the glass state are still unanswered: for example, does it represent a genuine thermodynamic phase of the matter or a purely kinetic phenomenon? How many "fictive" thermodynamic parameters are needed for characterizing the amorphous state? These and other questions are relevant not only for glasses but for every system with slow dynamics 2], such as dense granular materials and colloids, disordered dielectrics and electron glasses, etc.

Fifty years ago, Kovacs designed an experiment in which the low temperature behavior of glasses is probed by means of the following protocol [3] (see also [4, 5]). A sample is brought from high to low temperature $T_{1}$, and left to age for a time $t_{\mathrm{w}}$ until its volume $v$ attains the value it would have in thermal equilibrium at a temperature $T_{2}>T_{1}, v\left(t_{\mathrm{w}}\right)=v_{\mathrm{eq}}\left(T_{2}\right)$. The sample is then suddenly heated to $T_{2}$ and the ensuing volume relaxation is observed. Even though the average volume is already at its equilibrium value, it turns out that in order to fully equilibrate, the system first increases its volume, then passes through a maximum that depends on the thermal history of the system, and eventually recovers the equilibrium. The non-monotonic behavior generally re-

\footnotetext{
*Electronic address: arenzon@if.ufrgs.br

${ }^{\dagger}$ Research Associate at The Abdus Salam ICTP, Trieste, Italy

${ }^{\ddagger}$ Electronic address: sellitto@ictp.trieste.it
}

sults from the superposition of two antagonist processes: the fast modes - equilibrated at $T_{1}$, tend to increase the volume, while the slow modes - which are still relaxing towards $T_{1}$, tend to decrease it [5]. The sample is therefore formed by regions having an average local density larger or smaller than the equilibrium one, which respond on different time scales to a change in temperature. The experiment thus provides an indirect evidence that the low temperature dynamics of glasses is spatially heterogeneous $[3,4,5]$.

The Kovacs effect was first observed in polymeric glasses [3] and in statistical mechanical systems as simple as the ferromagnetic Ising chain [6]. There is an increasing interest in this phenomenon in connection with other kind of memory effects investigated in disordered magnets, and recent statistical mechanical approaches to glassy and granular matter. In these contexts, it has been observed that the Kovacs effect occurs in systems with quasi-long range order 7], spin glasses 8], realistic model of molecular liquid (ortho-terphenyl) 9|, traps models [10], kinetically constrained spin chains 11], and in simple models of granular compaction, such as the parking lot model [12]. More recently, the effect has also been studied in the spherical $p$-spin glass 13.

In this paper we study the Kovacs effect in facilitated $f$-spin models of glassy dynamics in spatial dimension $d$. Our interest here is mainly dictated by the possibility of using the Kovacs effect to identify the microscopic mechanism responsible for the slow relaxation (e.g., strong vs. fragile glass behavior [14]), or, more ambitiously, to discriminate between situations in which an arrest transition occurs from others in which it does not. We also investigate the inherent structure dynamics during the Kovacs protocol and show that models in spatial dimension $d>1$ and facilitation parameter $f>1$ are able to account for a subtle effect recently observed in molecular dynamics simulation of a realistic model of fragile glass forming liquid [9]. 


\section{Facilitated spin models}

The model we consider is model first introduced by Fredrickson and Andersen 15], with the purpose of describing the cooperative relaxation in highly viscous liquids. The spins are non interacting and the Hamiltonian density is simply

$$
e=\frac{1}{N} \sum_{i=1}^{N} s_{i}
$$

where the $s_{i}=0,1$ are spin variables, and the index $i$ runs over the sites of a lattice with coordination number $c$. One possible interpretation of the spin variable is that its state represents, after a suitable coarse-graining over molecular length and time scales, mesoscopic regions of the liquid with more or less density. What makes the model non trivial is the dynamics, which is defined by the following facilitation rule: $s_{i}$ can flip with a non-zero rate if and only if at least $f$ of its $c$ neighboring spins are up, that is

$$
h_{i} \equiv \sum_{k \mathrm{nn} i} s_{k} \geq f .
$$

The transition rates satisfy detailed balance and are proportional to the Metropolis factor

$$
\mathcal{W}\left(s_{i} \rightarrow 1-s_{i}\right)=\Theta\left(h_{i}-f\right) \min \left[1, \mathrm{e}^{\beta\left(2 s_{i}-1\right)}\right],
$$

where $\beta$ is the inverse temperature $\beta=1 / k_{\mathrm{B}} T$, and the Heaviside function $\Theta$ encodes the facilitation mechanism. It turns out that at low enough temperature the system dynamics is unable to relax to the equilibrium distribution within the simulation time window. In fact, as the density of up spins becomes smaller and smaller, the facilitation rule is hardly satisfied and consequently the spin relaxation becomes slower and slower. For this reason the model exhibits several glassy features, such as non-exponential relaxation, history dependent properties and heterogeneous distribution of local relaxation times [15, 16, 17, 18]. For a review see Refs. 19]. This type of facilitated spin model is reminiscent of the kinetically constrained lattice-gas introduced by Kob and Andersen 20]. However, the facilitated dynamics is not conserved and the constraint is weaker (as it involves only the local field $h_{i}$ of the spin which attempts to flip). Numerical investigations of two and three dimensional systems (with different values of $f>1$ ) suggested that the characteristic equilibration time at low temperature diverges as a Vogel-Fulcher law [17], although early arguments shown that the system is ergodic at any temperature larger than zero [16, 21]. More generally, the equilibration time has a non-Arrhenius behavior typical of fragile glasses for $d>1$ and $f>1$. The case $f=1$ instead corresponds to the defect diffusion dynamics and leads to the Arrhenius behavior, $\log t_{\mathrm{eq}} \sim 1 / T$, characterizing strong glasses. However, $1 d$ models, such as the
East model 23, 24] which have directed (or asymmetric) constraints, may also exhibit a non-Arrhenius behavior.

One reason of interest in the facilitated spin models is that they present many glassy features in spite of the absence of a true glass transition in finite dimension 21, 22], while on the Bethe lattice they appear to have an ergodic/non-ergodic transitions of the kind predicted by mode-coupling theory [25, 26, 27]. In the following we shall be interested in reproducing the Kovacs experiment in facilitated spin models defined on finite dimensional lattices with the purpose of comparing the behavior for different value of the facilitation parameter.

\section{Kovacs effect}

To numerically reproduce the Kovacs experiment an equilibrium configuration at high temperature is quenched at low temperature $T_{1}$ and left to age for a waiting time $t_{\mathrm{w}}$, until the energy density, $e$, attains the value it would have in thermal equilibrium at temperature $T_{2}>T_{1}, e\left(t_{\mathrm{w}}\right)=e_{\mathrm{eq}}\left(T_{2}\right)$. According to Eq. (1) the equilibrium energy density reads:

$$
e_{\mathrm{eq}}(T)=\frac{1}{1+\mathrm{e}^{\beta}} .
$$

The energy density therefore is the natural observable that plays in our model the role of the volume in the Kovacs protocol. This quenching procedure is repeated for several temperatures $T_{1}$. Each configuration obtained at time $t_{\mathrm{w}}$ such that $e\left(t_{\mathrm{w}}\right)=e_{\mathrm{eq}}\left(T_{2}\right)$, represents a distinct glassy state with different thermal history but identical energy. Such configurations are then quickly heated at temperature $T_{2}>T_{1}$, and the reduced energy $\Delta v$, defined as

$$
\Delta v\left(t, t_{\mathrm{w}}\right)=\frac{e(t)-e\left(t_{\mathrm{w}}\right)}{e\left(t_{\mathrm{w}}\right)},
$$

is recorded as a function of time $t\left(t>t_{\mathrm{w}}\right)$. We use a fast algorithm that keeps track of the list of free (unconstrained) spins, from which the attempted spin-flips are chosen with the usual Metropolis algorithm. Accordingly, the Monte Carlo time sweep is rescaled with the number of mobile spins. The largest number of spins we consider is $N=2^{21}$. The lattice systems have periodic boundary conditions.

In Fig. 1 (top) we show the reduced energy relaxation during a numerical experiment for a $3 d$ facilitated spin system with $f=3$, final temperature $T_{2}=1.0$ and several initial quenching temperatures $T_{1}$. Qualitatively similar results are obtained for $d=3$ and smaller facilitation parameters $(f=1,2)$, for the square lattice with $f=1,2$, as well as on a Bethe lattice (a random graph with fixed connectivity) where the glass transition occurs at a finite temperature for $f>1$ [27]. The plot exhibits the typical Kovacs hump very similar to those obtained in many other systems [6, 7, 8, 9, 10, 11, 12, 13], the hump 

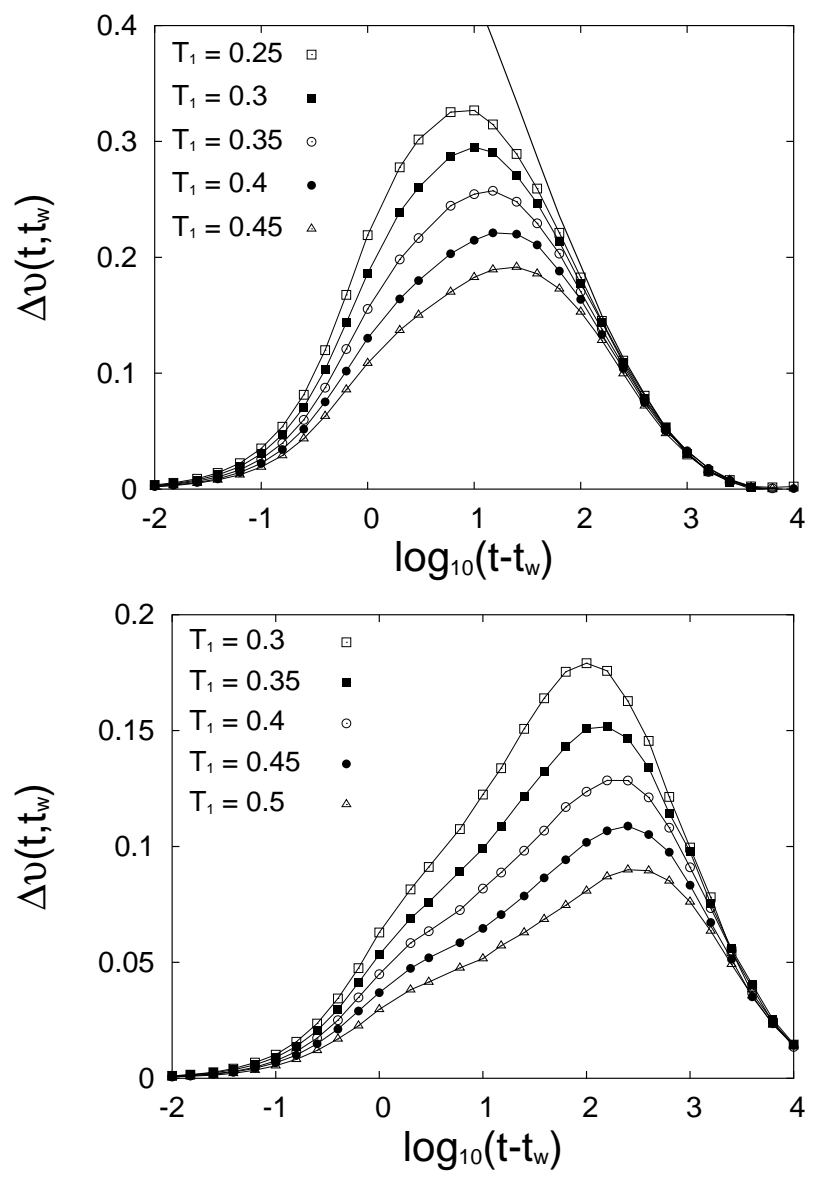

FIG. 1: Kovacs effect in a facilitated spin model on a cubic lattice with facilitation parameter $f=3$ (system size $V=128^{3}$ ). The system relaxes at temperature $T_{1}$ until it attains the energy $e\left(t_{\mathrm{w}}\right)=e_{\mathrm{eq}}\left(T_{2}\right)$; afterwards the temperature is changed from $T_{1}$ to $T_{2}=1.0$ (top) or 0.8 (bottom). The full line represents the energy relaxation after a quench at $T_{2}$. Qualitatively similar results are found for different dimensions, facilitations and geometries.

height increasing with $\Delta T=T_{2}-T_{1}$ and shifting to smaller times as $T_{1}$ decreases. By decreasing $\Delta T$ one ob-

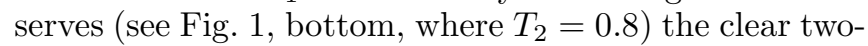
step behavior of the energy relaxation, as recently found in the $1 d$ case 11]. The fast, non activated processes due to the excitations of spins neighboring facilitated ones, contribute to the formation of a plateau starting on timescales [35] $t-t_{\mathrm{w}} \sim \mathcal{O}(1)$. On longer timescales, due to activated processes, the energy increases up to a maximum at time $t-t_{\mathrm{w}}=t_{\mathrm{K}}$, decreasing afterwards until the equilibrium is eventually attained. When rescaling time by a suitable characteristic time, $t / t^{*}$, and $\Delta v$ by the maximum height, $\Delta v_{\max }$, a good collapse of the late time regime is obtained, while deviations occur for the non activated part of the hump, as can be seen in Fig. 2 Usually $t^{*}$ has to be chosen much larger than the time of the maximum, $t_{\mathrm{K}}$, so that the contribution of the fast,

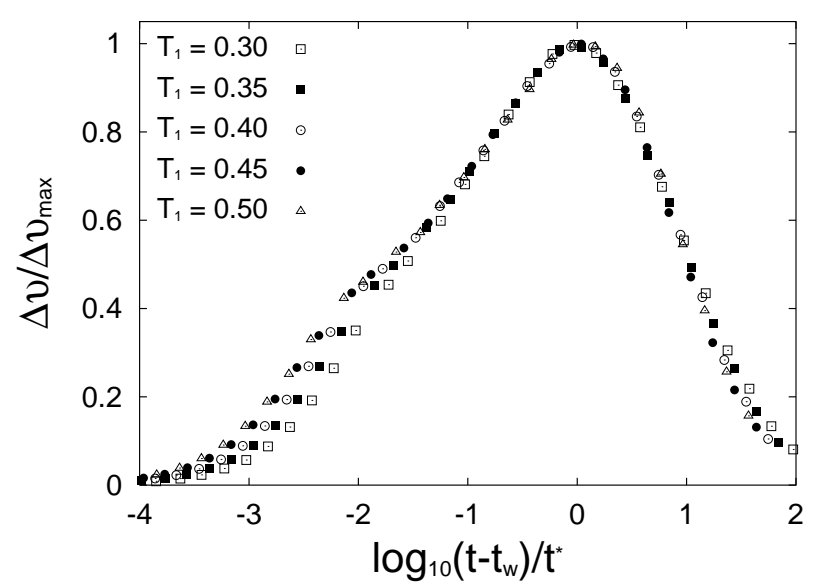

FIG. 2: Scaling of the data of Fig. 1 for $T_{2}=0.8$. Both time and height are rescaled, respectively by $t^{*}$ (see text) and $\Delta v_{\max }$. Data collapse is good except for the initial part where scaling is not expected to hold [8].

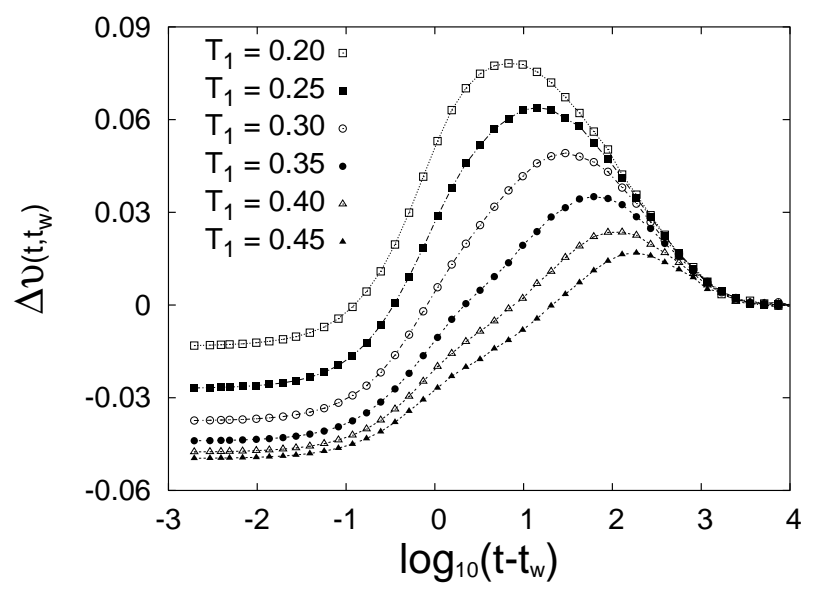

FIG. 3: Kovacs effect in a facilitated spin model on a cubic lattice with $f=3$ (system size $V=128^{3}$ ). The protocol here is different from fig. [1] the system relaxes at temperature $T_{1}$ for a time $t_{\mathrm{w}}=1000$ and then the temperature is changed from $T_{1}$ to $T_{2}=1$. See also Ref. [9].

non scaling modes vanishes [8]. Here, instead, as a consequence of the clear separation of time scales, $t^{*}$ is very close to $t_{\mathrm{K}}$.

Another possible way to investigate the Kovacs effect is to fix the time spent at temperature $T_{1}$ and then to change the temperature to $T_{2}$. An example is shown in Fig. 3 for $d=f=3$. This protocol has been used in Ref. [9]. One can notice that the results are qualitatively similar to those obtained with the previous protocol.

Finally, we must remark that, in order to remove the trivial part of the Kovacs effect, one should consider the initial time slightly after the jump as discussed in Ref. [10]. However, for the class of models considered 
here the contribution of fast modes, lasting for $\mathcal{O}(1) \mathrm{MCs}$, is clearly resolved due the presence of an intermediate plateau. Moreover, this initial increase can be removed by looking at the inherent structures dynamics. We also checked that within our protocol there is no Kovacs effect in the absence of kinetic constraints, confirming that the observed effects are not an artifact of our procedure.

\section{Temperature dependence of the Kovacs peak}

The results we have have obtained for different values of the facilitation parameter $f$, would lead to the conclusion that the Kovacs effect is unable to discriminate among the several microscopic mechanisms of slow relaxation or, even worse, between situations in which a dynamical arrest transition occurs from others in which it does not. As we show below, this is not the case: a more careful analyzes shows clear differences between all these cases. In particular, we have analyzed the behavior of the Kovacs peak time $t_{\mathrm{K}}$ as a function of the final temperature $T_{2}$ at fixed $T_{1}$, making a comparison with recent estimations of the equilibration times coming from independent methods.

For $d=1$ there are simple arguments 11] that correctly describe the dependence of both $t_{\mathrm{w}}$ and $t_{\mathrm{K}}$ on $T_{1}$ and $T_{2}$. The average distance between up spins is proportional to the inverse of their concentration, $e^{-1}(T) \sim$ $\exp (1 / T)$, for low $T$, while the effective diffusion rate for these spins is $\Gamma(T) \sim \exp (-1 / T)$. Thus, after the initial quench to $T_{1}$, the time $t_{\mathrm{w}}$ to attain the equilibrium concentration (at $T_{2}$ ) of up spins, $e_{\text {eq }}\left(T_{2}\right) \sim \exp \left(-1 / T_{2}\right)$, is $t_{\mathrm{w}}\left(T_{1}, T_{2}\right) \sim \Gamma^{-1}\left(T_{1}\right) e_{\mathrm{eq}}^{-2}\left(T_{2}\right) \sim \exp \left(1 / T_{1}+2 / T_{2}\right)$. When $T_{1}=T_{2}$ we obtain the equilibration time after a quench, $t_{\text {eq }} \sim \exp \left(3 / T_{2}\right)$. A similar argument, for the East model, leads to $t_{\text {eq }} \sim \exp \left(1 / T_{2}^{2} \log 2\right)$ and $t_{\mathrm{w}} \sim \exp \left(1 / T_{1} T_{2} \log 2\right)$. In both cases, it turns out that, as a function of $T_{2}, t_{\mathrm{K}} \sim t_{\text {eq. }}$. However, it is not clear whether simple arguments exist for other dimensions and facilitation parameters, although the correspondence between $t_{\mathrm{K}}$ and $t_{\mathrm{eq}}$ seems to remain valid, as is shown below.

Fig. 团 (top) shows $t_{\mathrm{K}}$ vs $T_{2}$ for the defect diffusion dynamics, $f=1$, and spatial dimension $d \leq 4$. In all cases, $t_{\mathrm{K}}$ shows an Arrhenius behavior in the limit $T_{2} \rightarrow T_{1}^{+}$and scales with $T_{2}$ as the equilibration relaxation time of the system. For $d=1$ we recover the result of Ref. 11], with $t_{\mathrm{K}} \sim \exp \left(3 / T_{2}\right)$. For higher dimensions, a generalization of the previous argument [19] gives $t_{\mathrm{eq}} \sim \exp \left[(1+2 / d) / T_{2}\right]$, and seems not to be correct (dashed lines in figure). Indeed, for $d=2$, the result $t_{\mathrm{K}} \sim$ $\exp \left(2.3 / T_{2}\right)$ is compatible with many, independent numerical estimates of $t_{\text {eq }}$, for example, from the integrated correlation function and the dynamical susceptibility 28 and from the distribution of persistence times [29]. This value is in the same universality class of directed percolation (DP) 30. For $d=3$ and 4 , a renormalization group analysis 31] gives $t_{\mathrm{eq}} \sim \exp \left[(2+\varepsilon / 12) / T_{2}\right]$, with $\varepsilon=4-d$, again in the same universality class of DP.
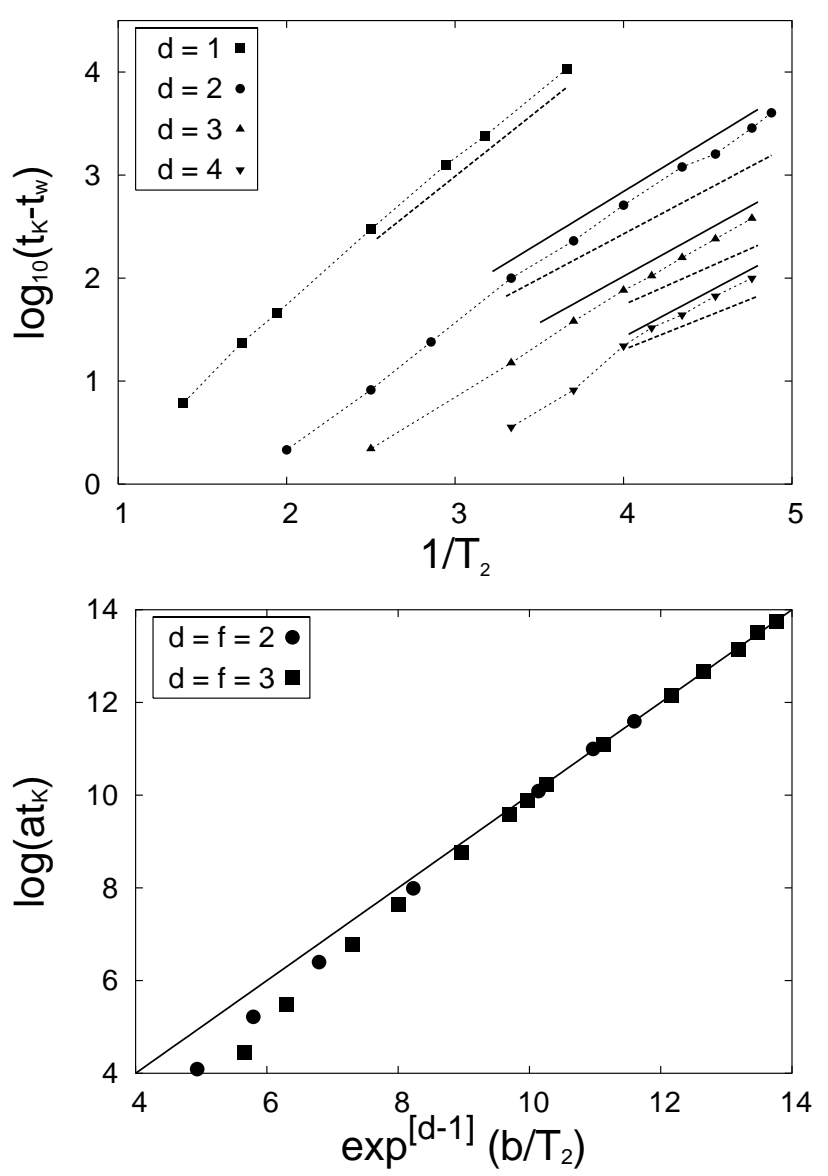

FIG. 4: Behavior of $t_{K}$ as a function of $T_{2}^{-1}$. Top: $f=1$ and $T_{1}=0.2$. In all cases the behavior, for $T_{2} \rightarrow T_{1}^{+}$ is Arrhenius. For comparison we show several predictions available in the literature. The solid lines correspond to: $t_{\mathrm{K}} \sim \exp \left(2.3 / T_{2}\right)\left[28,[29,32](d=2)\right.$ and $t_{\mathrm{K}} \sim \exp [(2+$ $\varepsilon / 12) / T_{2}$ ] 31] with $\varepsilon=4-d(d=3$ and 4$)$. The dashed lines correspond to the mean field result [11, 19]: $t_{\mathrm{K}} \sim$ $\exp \left[(1+2 / d) / T_{2}\right]$. Bottom: $d=f>1$, showing super Arrhenius behavior (see text). Notice the iterated exponential in the abscissa: for example, $\exp ^{[2]}(x) \equiv \exp (\exp (x))$.

In Fig. 4 (bottom) we show the results for the cooperative diffusion dynamics with $d=f>1$. In this case the systems exhibit a fragile glass behavior. Accordingly, we checked the Kovacs peak against an iterated exponential function $t_{\mathrm{K}} \sim \exp \left(\exp \left(b_{2} / T\right)\right)$ for $d=f=2$, and $t_{\mathrm{K}} \sim \exp \left(\exp \left(\exp \left(b_{3} / T\right)\right)\right)$ for $d=f=3[18,19,22,133]$. Again, our estimations agree pretty well, within the numerical accuracy, with those of the equilibrium relaxation time, obtained independently from the integral of the correlation function and the peak of the dynamical susceptibility [28]. For the East model, whose dynamics is also cooperative, again there is a correspondence with the relaxation time of the system, A first conclusion that we can draw from this analysis is that the Kovacs protocol is rather sensitive to the strong/fragile relaxation behavior and seems to provide an alternative, independent method 


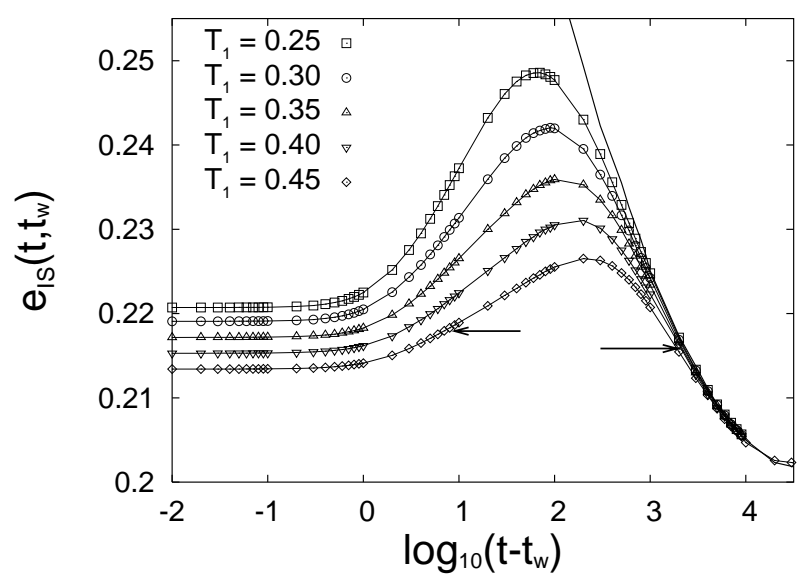

FIG. 5: Energy density of inherent structures during the Kovacs protocol for $d=f=3$ and several values of $T_{1}$. The lattice side is $L=64$ and $T_{2}=0.8$. The arrows show two ISs (with energy $e_{\mathrm{IS}}=0.2179$ and 0.2158 ) obtained by a zerotemperature descent from configurations having identical energy $e=0.24$ before and after the peak.

to obtain the equilibration time of the system.

Some extra information can also be obtained from $t_{\mathrm{w}}$, the time spent at $T_{1}$. In all cases we have considered here, the hump time, $t_{\mathrm{K}}$, and $t_{\mathrm{w}}\left(T_{1}, T_{2}\right)$, are related through a power-law $t_{\mathrm{K}} \sim t_{\mathrm{w}}^{b}$, where $b$ is temperature independent if $d=f$, and symmetric facilitation. On the other hand, for $d>f=1$ and in the East model, $b \sim 1 / T_{2}$ and $b \sim T_{2}$, respectively. In domain growth models one finds: $t_{\mathrm{K}} \sim t_{\mathrm{w}}^{1 / 2}(2 \mathrm{~d} \mathrm{XY}[7])$ and $t_{\mathrm{K}} \sim t_{\mathrm{w}}^{z /(1+z)}$ for the Ising model [10]. For $d=f=1$ we find $b \simeq 0.68$, close to the expected $2 / 3$ because in $1 d$ the dynamics proceeds as a domain growth process with dynamic exponent $z=2$ (we also obtain 2/3 from the expressions for $t_{\mathrm{K}}$ and $t_{\mathrm{w}}$ derived in Ref. 11] ). On the other hand, for $d=f=2$ and 3 we get $b \simeq 0.89$ and 1.05 , respectively, close to unity, as in the $p$-spin-glass model [13], where $b \simeq 0.9$.

\section{Inherent Structure Dynamics}

Finally, in order to make contact with recent results obtained by molecular dynamics simulation, Ref. [9], we investigated the inherent structure (IS) dynamics [34] during the Kovacs protocol. For spin models with stochastic dynamics such as those considered here, an IS is a blocked configuration, i.e. a configuration in which only isolated defects are present and no spin-flip is possible. The IS dynamics is obtained as follows: at every time $t$ a copy of the system is made and evolved with a zero-temperature sequential dynamics until it reaches a configuration in which no further spin-flip is possible. The energy $e_{\mathrm{IS}}$ of this IS is recorded and the dynamics of the original systems is resumed, this procedure being repeated along the system evolution. Fig. [5 shows a typical example of IS dynamics for $d=f=3$. First, one observes that even if the energies at time $t_{\mathrm{w}}$ are the same for all $T_{1}$, $e\left(t_{\mathrm{w}}\right)=e_{\mathrm{eq}}\left(T_{2}\right)$, the corresponding inherent structures are different: the lower is $T_{1}$, the higher is $e_{\mathrm{IS}}\left(t_{\mathrm{w}}\right)$. For times up to $\mathcal{O}(1)$, the IS evolution is confined in the same "energy basin" since the fast thermal excitations are removed by the zero-temperature descent. On longer time scales, the slow, activated processes start to play a role, the system is able to overcome the energy barriers and leave the initial basin, given origin to the hump. After the hump, the curves merge with the one obtained from a direct quench to $T_{2}$ (thick line in Fig. 沾) and eventually reach a value different (lower) than the initial one, that only depends on the equilibrium energy, $e_{\mathrm{IS}}\left(e_{\text {eq }}\right)$ (e.g., for $\left.f=1, e_{\mathrm{IS}}\left(e_{\mathrm{eq}}\right)=e_{\mathrm{ea}}\left(1-e_{\mathrm{eq}}\right)^{d}[36]\right)$. Thus, in analogy with the results of ref. 9], here too the IS explored far from equilibrium differ in depth from those sampled close to equilibrium. This difference increases with $T_{2}-T_{1}$ and is due to a different number of excitations, or equivalently, to a different realization of spatial heterogeneities, and not to topological properties of distinct regions of the phase space.

Once the system leaves the initial IS, two different behaviors are possible when one considers a couple of ISs descending from configurations having the same energy before and after the peak. While in the simplest case the two ISs may have the same energy there could be a more complicated case for which this is not true. The latter case has been observed recently by Mossa and Sciortino in molecular dynamics simulation of orto-terphenyl [9], a typical fragile glass forming liquid. We find that for $f>1$ there is an IS energy-shift similar to that observed in Ref. [9] (see Fig. 6] top, and the arrows in Fig. [5). For 1-spin facilitated dynamics instead, the ISs have the same energy (see Fig. [6] bottom, for an example). The reason of this distinct behavior does not seem directly related to the form of the relaxation time, but rather to the value of the facilitation parameter $f$. In fact, we find that in the East model, whose relaxation time is superArrhenius, no IS energy-shift is observed either [37]. Our results seem to suggest that only facilitated spin models with $d>1$ and $f>1$ are able to reproduce the subtle effect observed in Ref. [9].

In Fig. 6 we also show the IS sampled in equilibrium (thick lines) and, interestingly, in all cases, during the whole protocol the system explores regions never visited while in equilibrium: only asymptotically the points merges with the equilibrium curve. However, for smaller temperatures, the separation between the lower branch and the equilibrium curve diminishes, as well as the difference between the branches. Notice that in Ref. [9], soon after the hump, the system starts to sample configurations from a region of the phase space that is explored in equilibrium (but at different temperatures). 

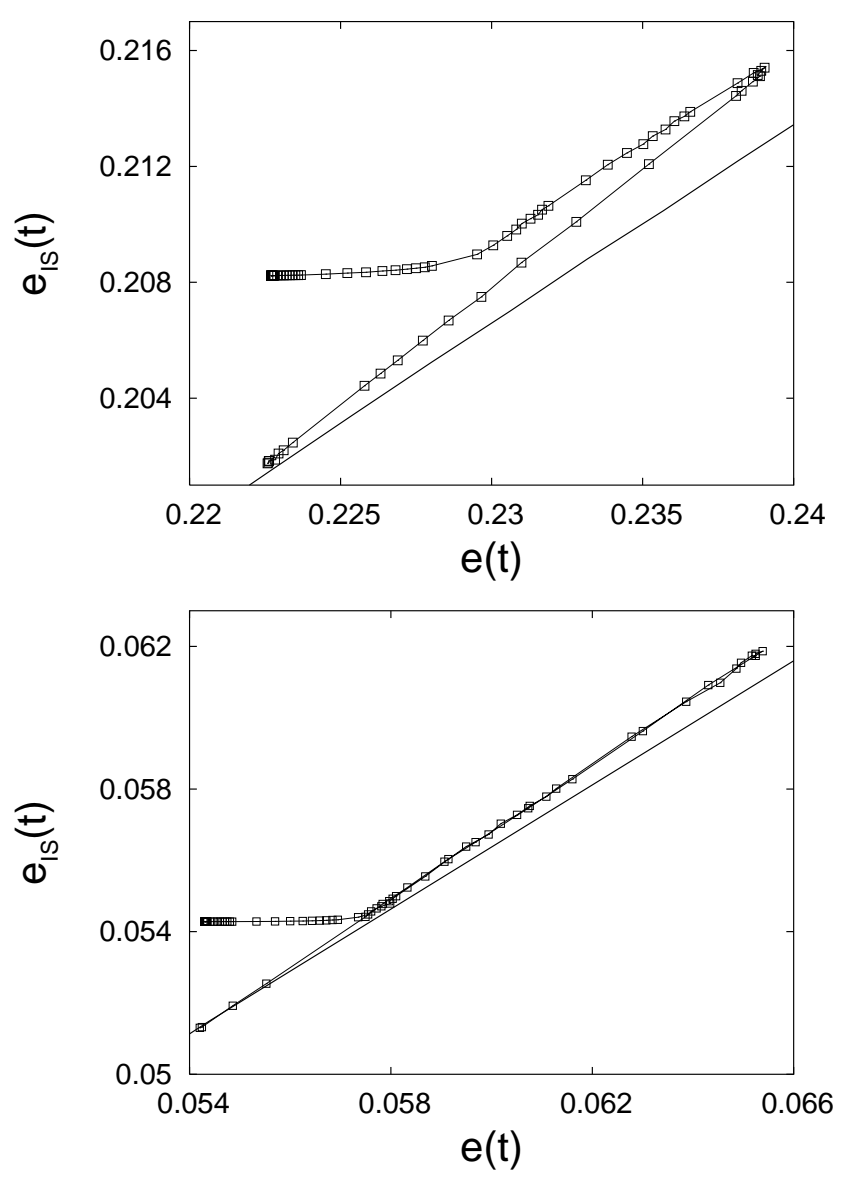

FIG. 6: Energy density of inherent structures during the Kovacs protocol versus the energy density. Top: $d=f=3$ for $T_{1}=0.6$ and $T_{2}=0.8$. The thick line is the equilibrium value, and both curves merge only at the end of the Kovacs protocol. Bottom: $d=f=1$ for $T_{1}=0.4$ and $T_{2}=0.7$. Notice that in this case, there is no difference between configurations with the same energy on both sides of the hump. For late times the curve merges with the equilibrium one (thick line), $e_{\mathrm{IS}}^{\mathrm{eq}}=e_{\mathrm{eq}}\left(1-e_{\mathrm{eq}}\right)^{d}$.

\section{Conclusions}

In summary, we have studied the Kovacs effect in a class of spin models with facilitated dynamics which ex- hibits slow relaxation at low temperature. Our results show that the Kovacs effect is qualitatively similar in all cases examined, irrespective of the spatial dimension the facilitation parameter, and the presence or not of a dynamic transition. However, in the class of facilitated models considered here one may distinguish between these situations by looking at temperature (of the final quench, $T_{2}$ ) dependence of the Kovacs peak time, which scales in the same way as the equilibrium relaxation time of the system at that temperature. Thus, the Kovacs protocol provides an independent way to gain information about the nature of the relaxation dynamics, at least for this class of models. It would be interesting to understand to what extent such a correspondence holds in more realistic systems, or in systems where the structural relaxation is related with the topography of the underlying potential energy landscape.

A related question concerns the inherent structures visited while the system follows the Kovacs protocol. Generally, they show the non trivial, activated character of the Kovacs effect, as well as the role played by spatial heterogeneities. In contrast with Ref. 9], the IS visited by the facilitated models considered here are not the equilibrium ones, which are only attained asymptotically. Moreover, the East and facilitated 1-spin models are not able to reproduce the subtle IS energy-shift observed in Ref. 9], while such an effect occurs in facilitated spin models with $d>1$ and $f>1$. This suggests that the inherent structure dynamics underlying the Kovacs protocol and the IS energy-shift might be used as a tool for discriminating between distinct microscopic mechanisms of dynamic facilitation. It would be also interesting to check by molecular dynamics simulation whether similar results hold for realistic models of strong and fragile glasses. This could provide a more stringent test of different scenarios of glassy dynamics.

\section{Acknowledgments}

JJA acknowledges the ICTP for support and hospitality, the Brazilian agencies CNPq and FAPERGS for partial support, and R.M.C. de Almeida, J.P. Garrahan, Y. Levin and D.A. Stariolo for useful conversations.
[1] G.B. McKenna, in Comprehensive Polymer Science, Vol. 2 Polymer Properties, edited by C. Booth and C. Price (Pergamon, Oxford, 1989), pp. 311-362.

[2] For a review, see: L.F. Cugliandolo, in Slow Relaxation and Nonequilibrium Dynamics in Condensed Matter, edited by J.L. Barrat, M. Feigelman, J. Kurchan, and J. Dalibard (Springer-Verlag, Berlin, 2003).

[3] A.J. Kovacs, Thèse, Fac. Sci. Paris (1954), and Adv. Polym. Sci. 3, 394 (1963).

[4] L.C.E. Struik, Physical Aging in Amorphous Polymers and Other materials, (Elsevier, Houston, 1978), Chap. 9.

[5] See, for example: R.G. Larson, The Structure and Rheology of Complex Fluids, (Oxford University Press, Oxford, 1999), Chap. 4, and references therein.

[6] S.A. Brawer, Phys. and Chem. of Glasses 19, 48 (1978).

[7] L. Berthier and P.C.W. Holdsworth, Europhys. Lett. 58, 35 (2002).

[8] L. Berthier and J.-P. Bouchaud, Phys. Rev. B 66, 054404 (2002).

[9] S. Mossa and F. Sciortino, Phys. Rev. Lett. 92, 045504 
(2004).

[10] E.M. Bertin, J.-P. Bouchaud, J.-M. Drouffe and C. Godrèche, J. Phys. A: Math. Gen. 36, 10701 (2003).

[11] A. Buhot, J. Phys. A: Math. Gen. 36, 12367 (2003).

[12] G. Tarjus and P. Viot, preprint cond-mat/0310738

[13] L.F. Cugliandolo, G. Lozano and H. Lozza, Eur. Phys. J. B 41, 87 (2004).

[14] C.A. Angell, Science 267, 1924 (1995).

[15] G.H. Fredrickson and H.C. Andersen, Phys. Rev. Lett. 53, 1244 (1984).

[16] G.H. Fredrickson and S.A. Brawer, J. Chem. Phys. 84, 3351 (1986).

[17] I.S. Graham, L. Piché, and M. Grant, Phys. Rev. E 55, 2132 (1997).

[18] S. Butler and P. Harrowell, J. Chem. Phys. 95, 4454 (1991).

[19] F. Ritort and P. Sollich, Adv. Phys. 52, 219 (2003).

[20] W. Kob and H.C. Andersen, Phys. Rev. E 48, 4364 (1993).

[21] J. Reiter, J. Chem. Phys. 95, 544 (1991).

[22] C. Toninelli, G. Biroli and D. Fisher, Phys. Rev. Lett. 92, 185504 (2004).

[23] J. Jäckle and S. Eisinger, Z. Phys. B 84, 115 (1991)

[24] P. Sollich and M.R. Evans, Phys. Rev. Lett. 83, 3238 (1999).

[25] J. Reiter, F. Mauch and J. Jäckle Physica A 184458 (1992).
[26] S.J. Pitts, T. Young and H.C. Andersen J. Chem. Phys. 1138671 (2000)

[27] M. Sellitto, G. Biroli and C. Toninelli, preprint cond-mat/0409393

[28] M. Sellitto, unpublished

[29] J.J. Arenzon, unpublished.

[30] H. Hinrichsen, Adv. Phys. 49, 815 (2000).

[31] S. Whitelam, L. Berthier and J.P. Garrahan, Phys. Rev. Lett. 92, 185705 (2004).

[32] J.P. Garrahan, private communication

[33] Jäckle J., J. Phys.: Cond. Matter 14, 1423 (2002).

[34] F.H. Stillinger and T.A. Weber, Phys. Rev. A 28, 2408 (1983).

[35] In $1 d$, this corresponds to the nucleation of smaller clusters inside the previously formed domains, defined as a sequence of contiguous down spins, plus the left up spin [24]. A simple mean field argument taking this into account, is able to correctly describe both the linear behavior at short times and the height of the plateau.

[36] In $d=1$, as one sequentially sweeps the lattice, every up spin that has an up spin to its right (those at left were already flipped down) will become 0 . The remaining up spins, occurring with probability $e_{\mathrm{eq}}\left(1-e_{\mathrm{eq}}\right)$, contribute to $e_{\mathrm{IS}}$. This is simply generalized in higher dimensions.

[37] We also mention that the IS energy-shift occurs in directed models in higher dimensions, such as the NorthEast model. 